\title{
Roadroid: Continuous Road Condition Monitoring with Smart Phones
}

\author{
Lars Forslöf ${ }^{1}$ and Hans Jones ${ }^{1,2}$ \\ 1. Roadroid AB, Ljusdal 82735, Sweden \\ 2. Department of Computer Engineering, Dalarna University, Borlänge 78450, Sweden
}

\begin{abstract}
Road condition is an important variable to measure in order to decrease road and vehicle operating/maintenance costs, but also to increase ride comfort and traffic safety. By using the built-in vibration sensor in smart phones, it is possible to collect road roughness data which can be an indicator of road condition up to a level of Class 2 or 3 in a simple and cost efficient way. Since data collection therefore is possible to be done more frequently, one can better monitor roughness changes over time. The continuous data collection can also give early warnings of changes and damage, enable new ways to work in the operational road maintenance management, and can serve as a guide for more accurate surveys for strategic asset management and pavement planning. Collected measurement data are wirelessly transferred by the operator when needed via a web service to an internet mapping server with spatial filtering functions. The measured data can be aggregated in preferred sections, as well as exported to other GIS (geographical information systems) or road management systems. Our conclusion is that measuring roads with smart phones can provide an efficient, scalable, and cost-effective way for road organizations to deliver road condition data.
\end{abstract}

Key words: Road condition, asset management, mobility, smart phone, intelligent transportation systems.

\section{Introduction}

The IRI (international roughness index) [1] is a road roughness index commonly obtained from measured longitudinal road profiles. Since its introduction in 1986, the IRI standard has become commonly used worldwide for evaluating and managing road systems [2]. However, measuring road roughness has been used since early 1900s for expressing road condition and ride quality [3]. Since the end of the 1960s, however, most road profiling is done with high speed road profiling instruments [2].

The modern traditional techniques for measuring roughness may be categorized as either specially built trucks or wagons with laser scanners, bump-wagons or even manually operated rolling straight edges. Specially built measurement equipment is expensive, due to heavy and complex hardware, low volume of

Corresponding author: Lars Forslöf, B.Sc., CEO-founder, research fields: intelligent transportation system, mobile surveying and traffic management. E-mail: lars.forslof@roadroid.com. production and the need of sophisticated systems and accessories.

Data gathering and analysis are often time consuming. In the northern hemisphere, data collection is typically done during the summer, then analyzed and delivered to the maintenance management systems in late autumn. During the winter and spring, the road usually faces a continual frost heave/thaw (a very dramatic period in a road's life with extreme changes in roughness) cycle. The IRI values that were "exact" almost a year ago may now not be the same any longer. Besides, since it is so expensive to collect and analyze the data, many roads are only covered in one lane direction every 3 rd or 4 th year.

Smart phone based gathering of roughness data which essentially are a RTRRMS (response-type road roughness measuring system) [1] can be done at a low cost and monitor changes on a daily basis. For frost and heave issues, it can tell when and where it is happening and if the situation is worse than in previous years. It can also be used in the winter to determine the 
performance of snow-removal and ice-grading. It may be advantageously used in performance based contracts or research on road deterioration, various environmental effects (as heavy rains, flooding, etc.) and other adjacent purposes.

It should be mentioned that smart phone based systems like Roadroid might challenge old road knowledge with regard to standards, procedures and existing ways to procure:

- pavement planners and road engineers knowledge of existing solutions and inputs;

- research organizations, suppliers and buyers who have established existing ways to work;

- organizations which have invested time, prestige and huge amounts of money to develop complex data collection and management systems which can present a very exact result.

As described in Ref. [1], it is necessary to understand the difference between the four generic classes of road roughness measuring methods in use:

- Class 1-precision profiles;

- Class 2-other profilometric methods;

- Class 3-IRI estimates from correlation equations;

- Class 4-subjective ratings and uncalibrated measures.

Data collection with smart phones will not directly compete with Class 1 [1] precision profiles measurements, but instead, complement them in a powerful way. As Class 1 data are very expensive to collect, it cannot be done often. Beside this, advanced data collection systems also demand complex data analysis and take long time to deliver the result. With smart phone based data collection, it is possible to meet both these challenges. A smart phone based system is also an alternative to Class 4-subjective rating [1], on roads where heavy, complex and expensive equipment is impossible to use, and for bicycle roads. The technology is objective, highly portable, and is simple to use. This gives a powerful support to road inventories, inception reports, tactical planning, program analysis and support maintenance project evaluation.

On the other end of the scale, many road inventories and assessments are made by humans (Class 4 subjective ratings) over large areas using only pen and paper. Smart phone roughness data collection fills a gap between Class 1 measurements and Class 4 visual inspections.

The Roadroid smart phone solution has two options for roughness data calculation:

(1) eIRI (estimated IRI) — based on a peak and RMS (root mean square) vibration analysis-which is correlated to Swedish laser measurements on paved roads. The setup is fixed but made for three types of cars and is thought to compensate for speed between $20 \sim 100 \mathrm{~km} / \mathrm{h}$. eIRI is the base for the RI (Roadroid index) classification of single points and stretches (road links) of the road;

(2) cIRI (calculated IRI) - based on the QCS (quarter-car simulation) [1] for sampling during a narrow speed range such as $60 \sim 80 \mathrm{~km} / \mathrm{h}$. When measuring cIRI, the sensitivity of the device can be calibrated by the operator to a known reference.

\section{The First Prototypes 2002-2006}

The Roadroid team has been working with mobile ITS since the mid-1990s, particularly with mobile data gathering, road weather information and road databases. During a visit to the Transportation Research Board in Washington in 2001, a Canadian project was presented that monitored the speed of timber hauling trucks. It simply assumed that if the speed was low, the road quality was poor. The idea was to add vibration measurements.

Together with the Royal Institute of Technology, a first pilot scheme was built in 2002-2003. At that time, we used a high-resolution accelerometer at the rear axle of a front wheel drive vehicle, connected by cable to a portable PC (personal computer) through a signal conditioner. Two master students built a first prototype using an industrial software system for signal analysis. 
The initial results were promising and the SNRA (Swedish National Road Administration) financed a R\&D (research and development) project to further develop and validate the prototype with a focus on gravel roads. The system was developed for an embedded Windows car PC with external GPS (global positioning system) and GSM (global system for mobile communications) capabilities as well as a special A/D (analogue/digital) board connected to the accelerometer. Also, a client and web based GIS (geographical information systems) tool was implemented for viewing the road quality spatially in different colors.

A validation between visual inspections and the systems measurements was performed and presented at the transport forum in Linköping in 2005.

This research was based on 35 segments of $100 \mathrm{~m}$ which were individually assessed according to four road condition classes. A MATLAB module analysis (experimental analysis of oscillation) was performed on reference samples of specific sections of the four condition classes.

A regression analysis was then performed with rules based on:

(1) accelerometer amplitude levels;

(2) RMS (root mean square) algorithms;

(3) measured vehicle speed;

(4) sample data length.

The analysis showed that a single test run with the

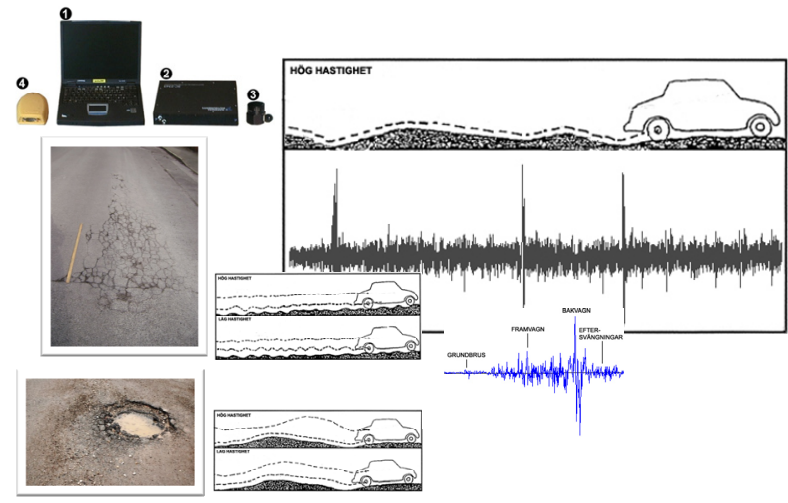

(a) system properly could classify up to $70 \%$ correct compared to an average of subjective visual expert inspections. A single subjective visual expert inspection could however vary much more from the average correct than the systems classification. The method was considered objective with very good repeatability (Fig. 1).

In 2006, the development stalled. The system was considered relatively cheap and simple to operate at the time. In retrospect, it had several limitations, particularly the sensor mounting and cables exposed in the harsh often wet environment under the vehicle chassis, also, the limitations from non-integrated standard components as Windows 98, the car PC, specific cable connections, and to handle the system solution as a whole by the end user.

\section{Further Development 2010-2011}

In 2010, the ideas from 2002-2006 were reviewed. A major technical development was the appearance of smart phones. Literally, all peripherals that previously were connected by special cables were now built into a smart phone and the limitations of certain components were removed by new advances in technology. We knew the answers to some of the questions from 2002-2006, e.g., the basis for signal analysis, the influence of speed and different vehicle characteristics, etc.. There were however new big questions to solve, such as:

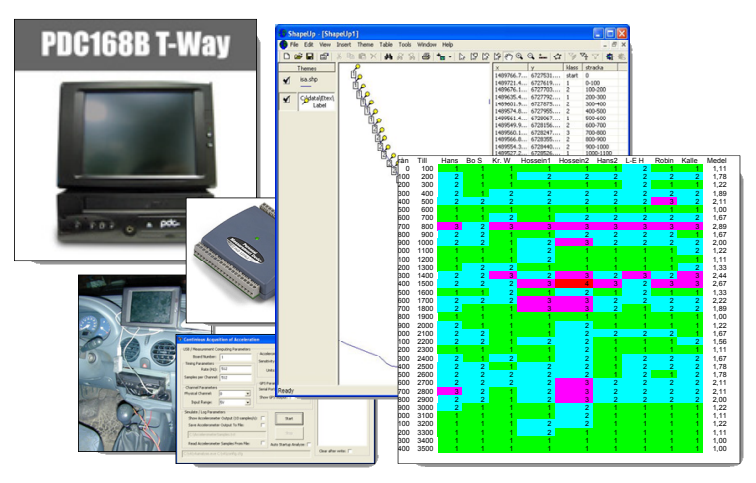

(b)

Fig. 1 Images from the history: (a) 1st prototype 2002-2003; (b) 2nd prototype, developed from 2004-2006. Source: Roadroid internal documents. 
- Was it possible to pick up the "filtered" signals from the vehicle chassis?

- We knew different car models most certainly would give different signals, how could we handle this?

- Would a lower sampling frequency be enough (around $100 \mathrm{~Hz}$ compared to earlier 512 1,024 Hz)?

- Would the accelerometer sensitivity and range (usually $+/-2$ g) be sufficient in a smart phone?

- Would different smart phone models return different measuring values (mainly accelerometer sensitivity and sample frequency)?

We developed an Android application and some test algorithms using the built-in accelerometer signal. The choice of Android rather than iPhone was made considering the open architecture and hardware price/performance relation. We started to sample data on different roads with different types of vehicles, and run over constructed obstacles in 2011.

We choose the best hardware at the time as reference device (Samsung Galaxy Tab GT P1000). The obstacles were run over by different vehicle types (from small car to large 4WD jeep) a number of times in six different speeds: 20, 40, 60, 80, 100 and 120 $\mathrm{km} / \mathrm{h}$ (Fig. 2).

Data were sampled with different devices, both with our algorithms and by collecting the raw accelerometer signal. During the data analysis, we discovered a number of things:

- Differences between different car models,

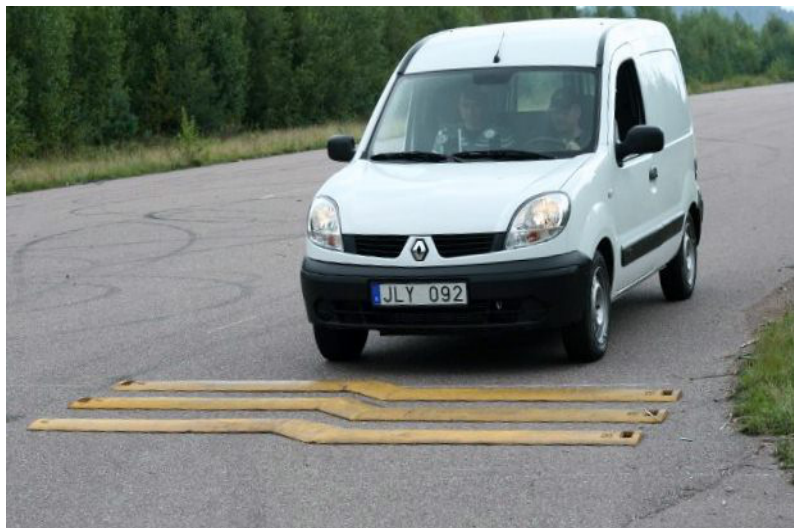

Fig. 2 Testing the 3rd prototype in 2011.

Source: Roadroid internal documents. especially at low speeds. In the $40 \sim 80 \mathrm{~km} / \mathrm{h}$ range, differences are however limited. The tests gave us a model for how to calculate the speed influence of the signal for three different types or classes of vehicle chassises;

- Discrepancies between different devices, both for the sampling frequency and the sensitivity of the accelerometer (up to $50 \%$ ). It is of great importance to know these dynamics to achieve comparable data. A device calibration procedure which can translate the unit characteristics to a known reference device is therefore required;

- It is important to mount the device firmly in a good mounting bracket, preferably in a way that enables the devices camera lens to be directed at the road. Unfortunately, few devices have good mounting brackets.

Most importantly, the trials (Fig. 2) during 2011 showed that usable data could be obtained.

\section{Viewing of Data}

Having a device delivering measurement data (no mobile network connection is needed during the sampling), we needed a suitable viewer of the information. We created a multi-user measurement viewer - an HTML5 based map tool to present the road condition data (Fig. 3). The data (which are encrypted) are compressed and sent directly from the device via a file transfer service (HTTP (hyper text transport protocol)

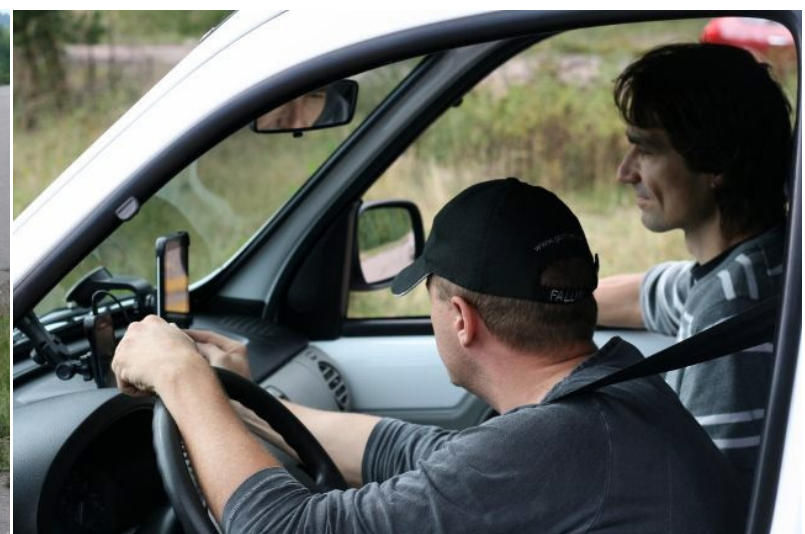




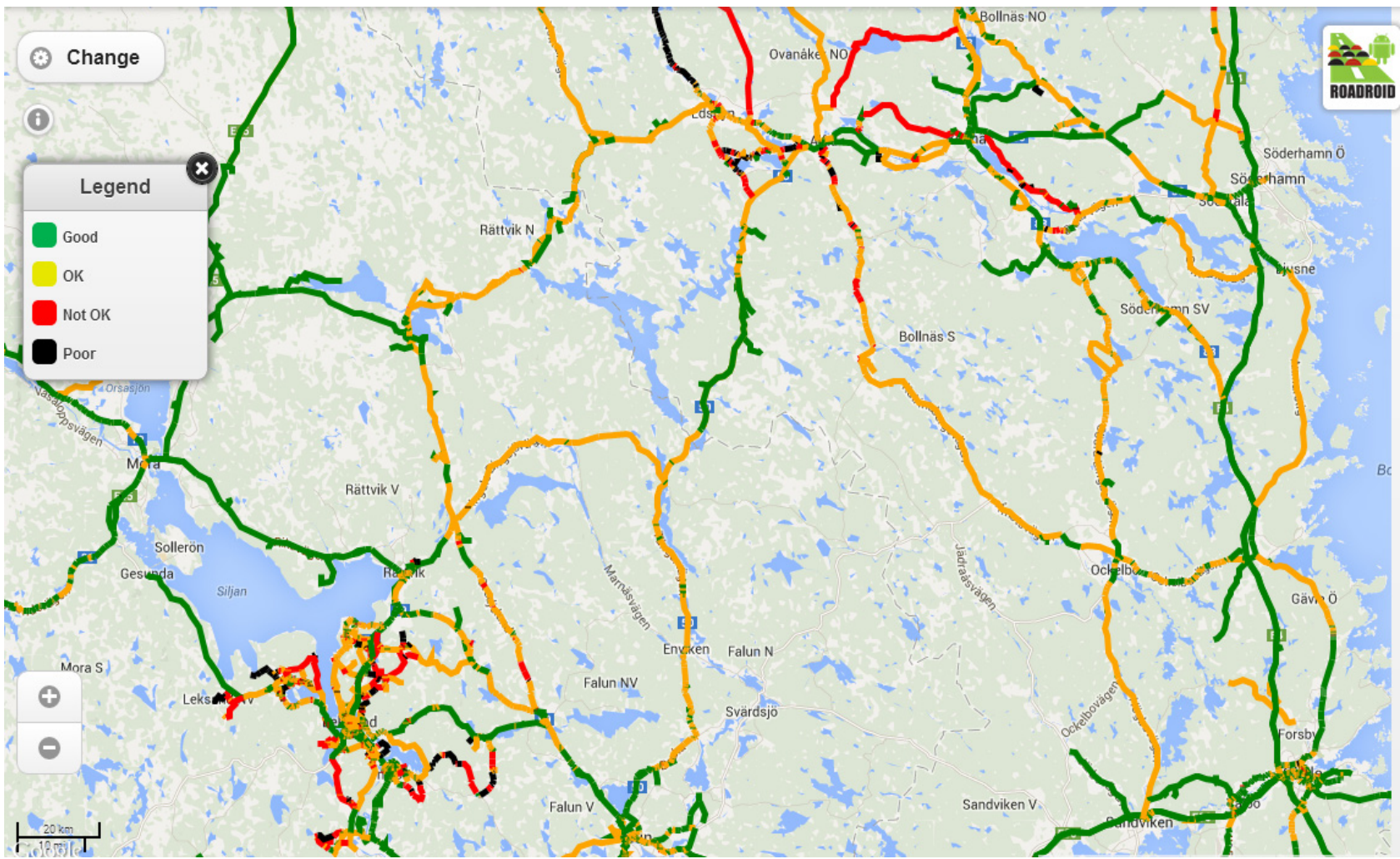

Fig. 3 A screenshot of the web GIS tool from the middle of Sweden.

Source: www.roadroid.com.

or FTP (file transfer protocol)) to our web server in the cloud. The uploaded data files from different units are by an hourly routine matched spatially and imported to road links/geometries such as OSM (open street map) [4], etc.. As background map, Google Maps with OpenLayers [5] is used to present the road condition data. The road condition data are divided into four different levels for visualization: green for good; yellow for satisfactory/OK; red for unsatisfactory/not $\mathrm{OK}$ and black for poor.

The mobile app stores a number of data values each second into a CSV (comma-separated value) file. But to get an overview on a larger scale, it is more convenient to use road links with aggregated and averaged measurements than individual sampled dots. Depending on the spatial road database, there will be many opportunities to refine the data and add attribute information such as road width, traffic volumes, etc.. In Sweden, we have been using the Swedish NVDB (national road data base) [6]. Globally, we have mostly been using geometries from OSM. The road condition data can be exported in shape format to other systems.

\section{Use of Data and the Roadroid Index}

We have been undertaking studies of the IRI and implemented the IRI computations [7] using the QCS for our cIRI value. According to Wakeham and Rideout [8], there is limited benefit of using the more complex half-car model since the half-car and quarter-car results were nearly identical.

We have also developed a correlation between our own road condition data algorithms and the IRI for our eIRI value. Furthermore, we continuously have been looking to improve the way we present the comprehensive level of information being collected - flexibility and scalability are the keys here. We wanted to be able to add data from several measurements over time and compare results over time in a flexible way. We also wanted to automatically generate reports for a specific road and to compare roads with each other and to do comparisons within whole regions. The solution was to use the percentage 
of each road class for the individual sampled dots which have been spatially connected to a road link or a geographic area (Fig. 4). We call this the RI. The RI is scalable for a part of a road, a whole road, a city, a region or even the whole world!

As we wanted to do continuous monitoring to view development over time, we also needed to find a way to produce reports. Data collection can be made by the contractor's road guards/officers who are doing visual inspections one to three times per week, or by other operators such as the local newspaper distributor. The RI seemed to be a suitable way to also make reports from the road condition data and trend changes over time (Fig. 5).

\section{Estimated IRI}

We now had a promising and scalable index, but also knew that we needed to correlate this to IRI. To find the correlation, we gathered:

- data from Class 1 (laser beam) IRI measurements, both in $20 \mathrm{~m}$ lengths and the data averaged to road link sections in NVDB [6];

- our average road condition values, both for the matching corresponding $20 \mathrm{~m}$ lengths and the whole road link sections in the NVDB [6].

By comparing hundreds of road link sections, we established a correlation factor and could now estimate an IRI value (eIRI). eIRI is usable all the way from individual sampled and classified dots with $1 \mathrm{~Hz}$ resolution to a complete road link average. The coefficient of determination $\left(R^{2}\right)$ was 0.5 (Fig. 6) which meant that it is moderately correlated.

We have noticed some limitations in speed adaptation, rough pavement surfaces and that minicars are quite more sensitive than our reference small car. Research is continuing by different institutions around the world, as the World Bank, UN OPS (Office for Project Services), specialized universities and some large road companies as SpeaAutostrade (Fig. 7). These organizations will report back to inform our development department, in order to improve our solution.

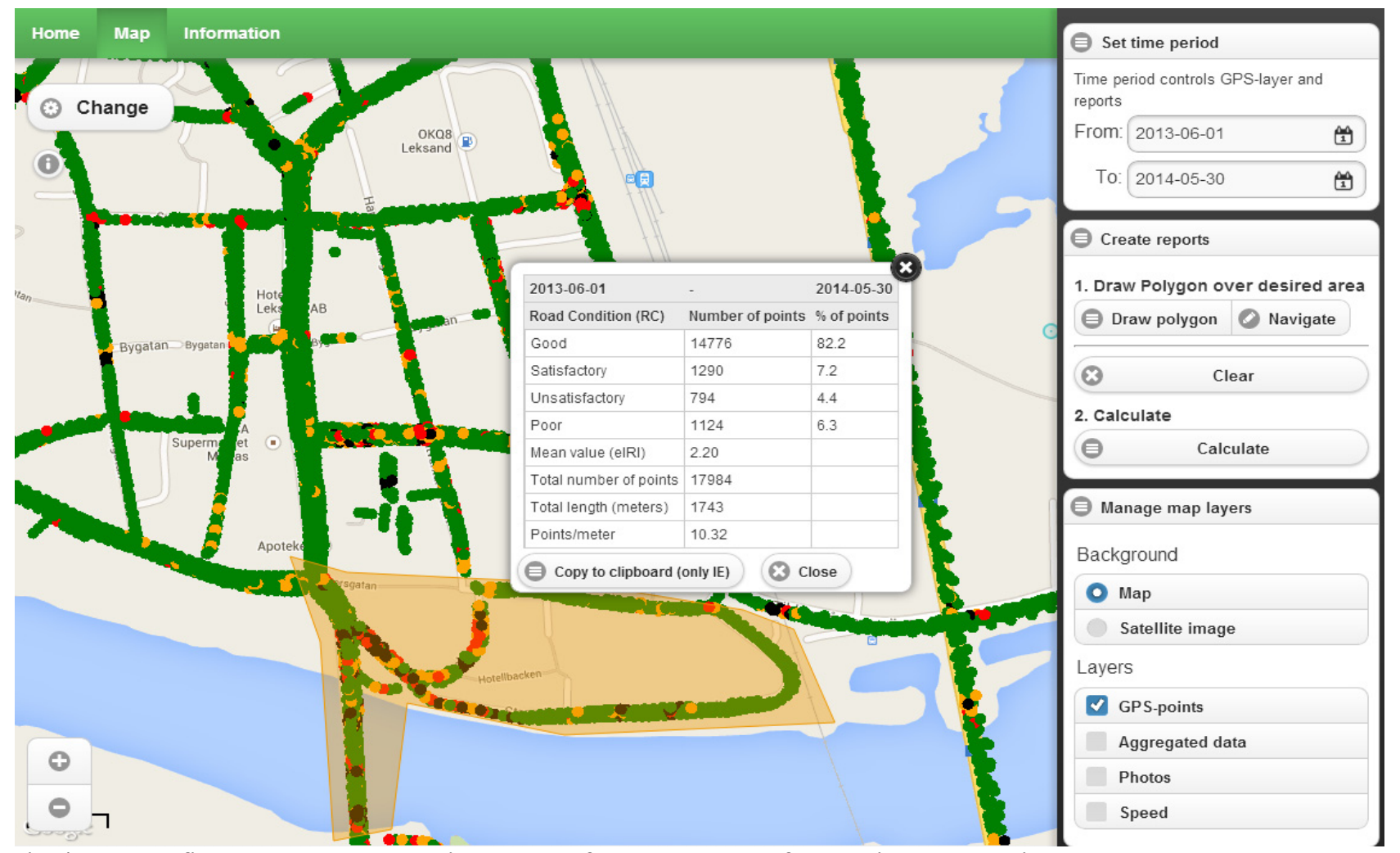

Fig. 4 How to filter, select layers and pick data out from the web tool for creating reports with the RI.

Source: www.roadroid.com. 


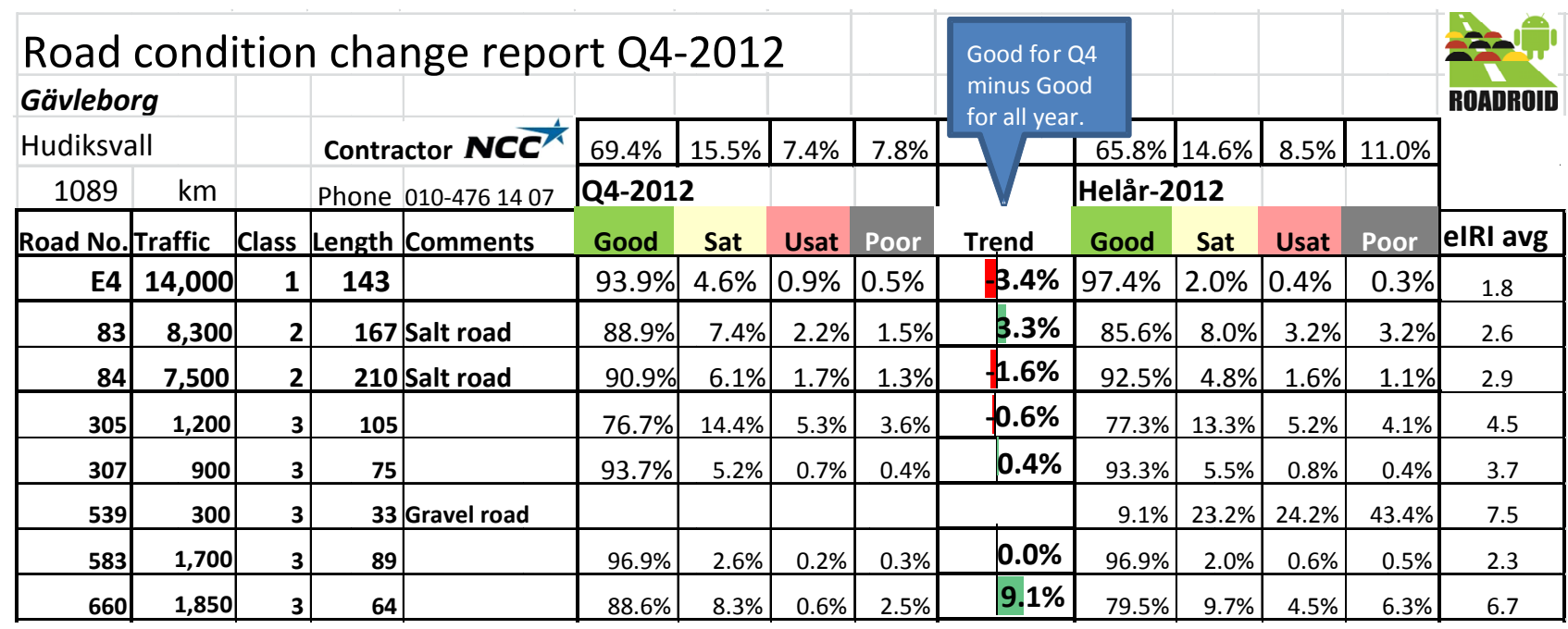

Fig. 5 A road condition change report using the RI-for performance follow up of the four conditions. The figure shows the condition changes for a specific road link during Quarter Four compared to the averaged whole year numbers.

Source: Roadroid internal customer documents.

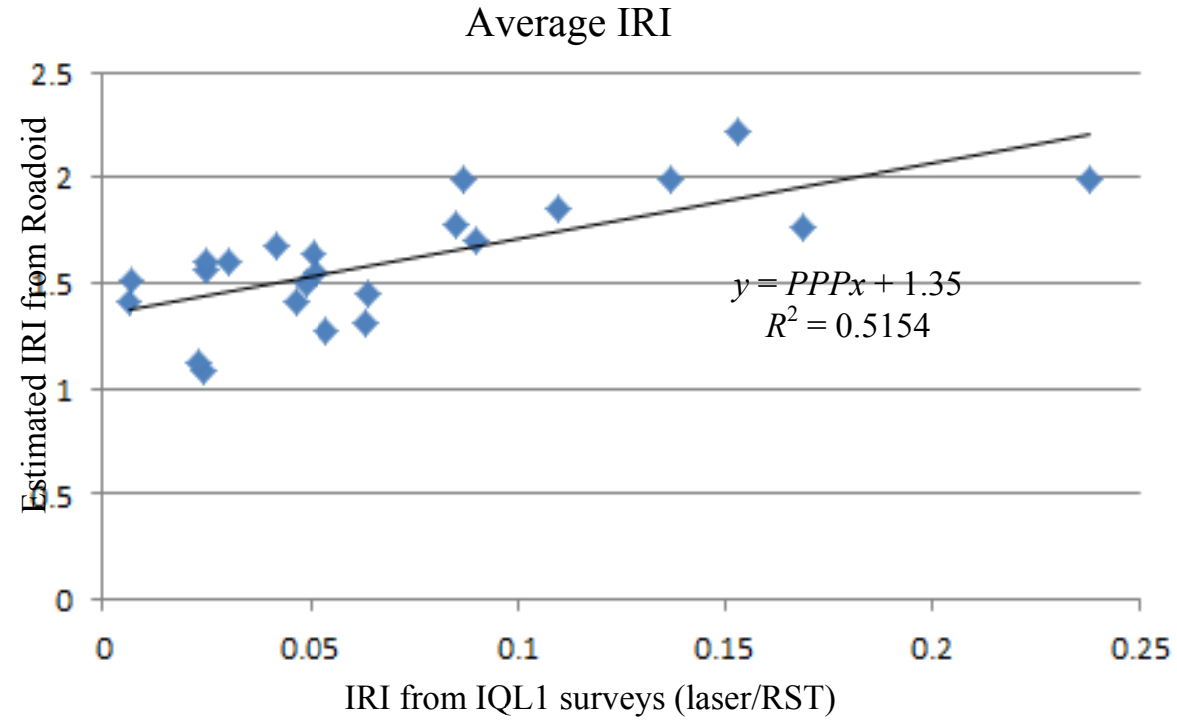

Fig. 6 From our base correlation study with eIRI.

Source: Roadroid internal documents.

Research done by the University of Pretoria in 2013 (Fig. 8) [9] was mainly concentrated on finding out if the Roadroid application is consistent enough during IRI measurements with varying vehicle conditions as: speed, road path, loads and tyre pressure, etc.. The stated conclusion was that Roadroid would be able to produce good results if the mentioned key indicators are standardized.

Research done by the University of Auckland in $2013[10,11]$ was mainly focused on if the Roadroid application could represent the roughness felt by motorists to a certain level. Both the reports found that the Roadroid application responded to the various road characteristics of the Auckland network in a similar manner to industry accepted systems. To be precise, Roadroid had an $81 \%$ correlation with laser measurement systems.

\section{Calculated IRI}

With feedback from organizations and by internal 


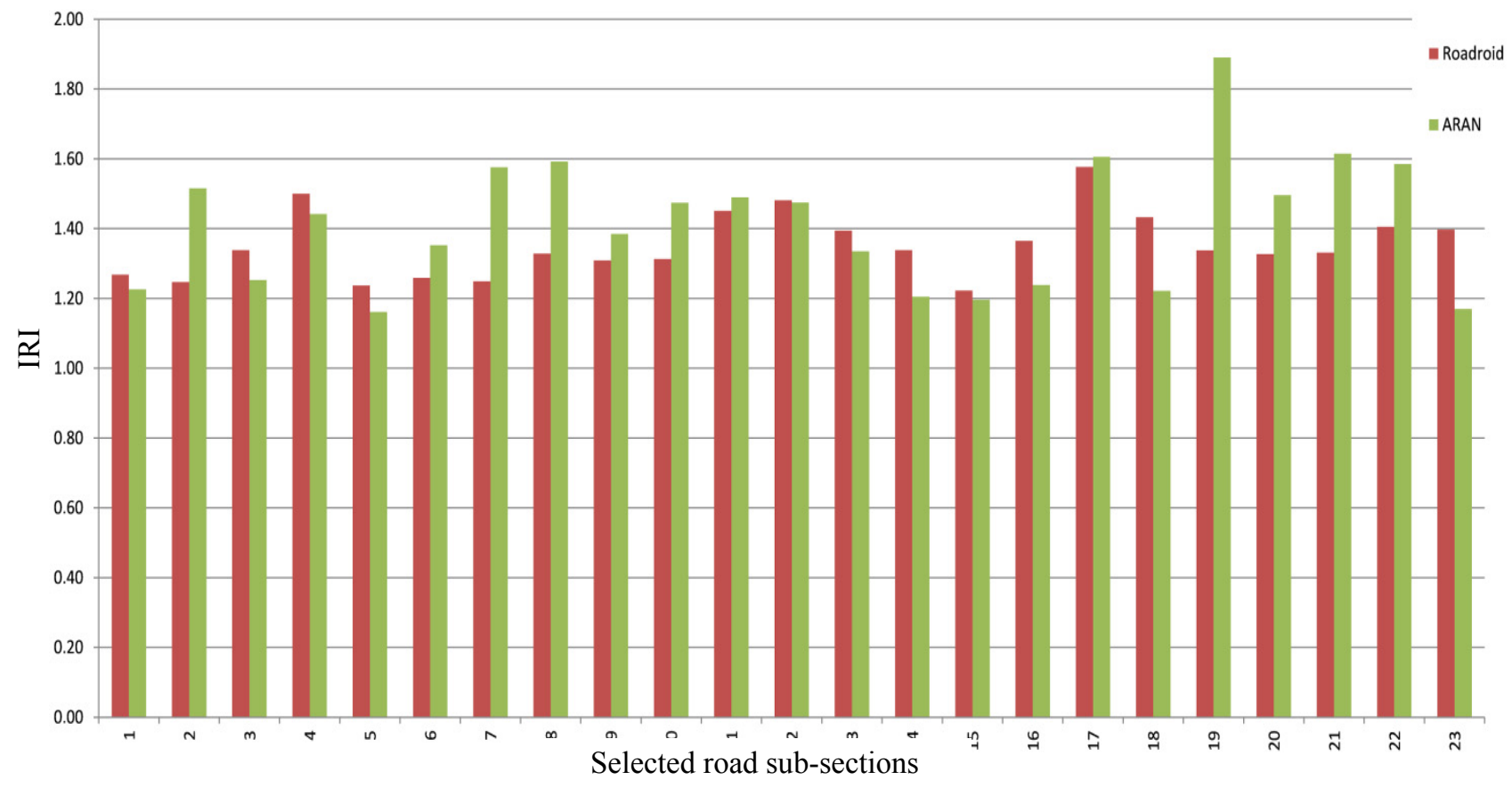

Fig. 7 IRI comparisons of cIRI (red) vs. ARAN (autostrade Aran IRI reference, green) on selected road sub-sections. Source: Roadroid internal documents.

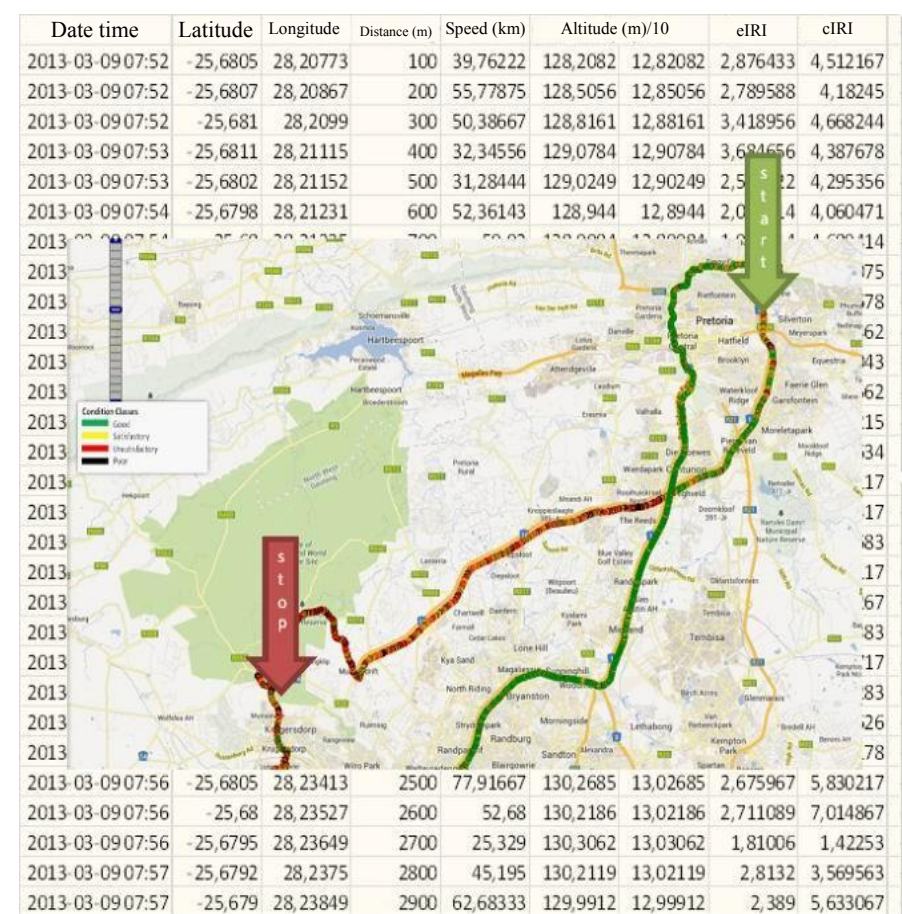

(a)
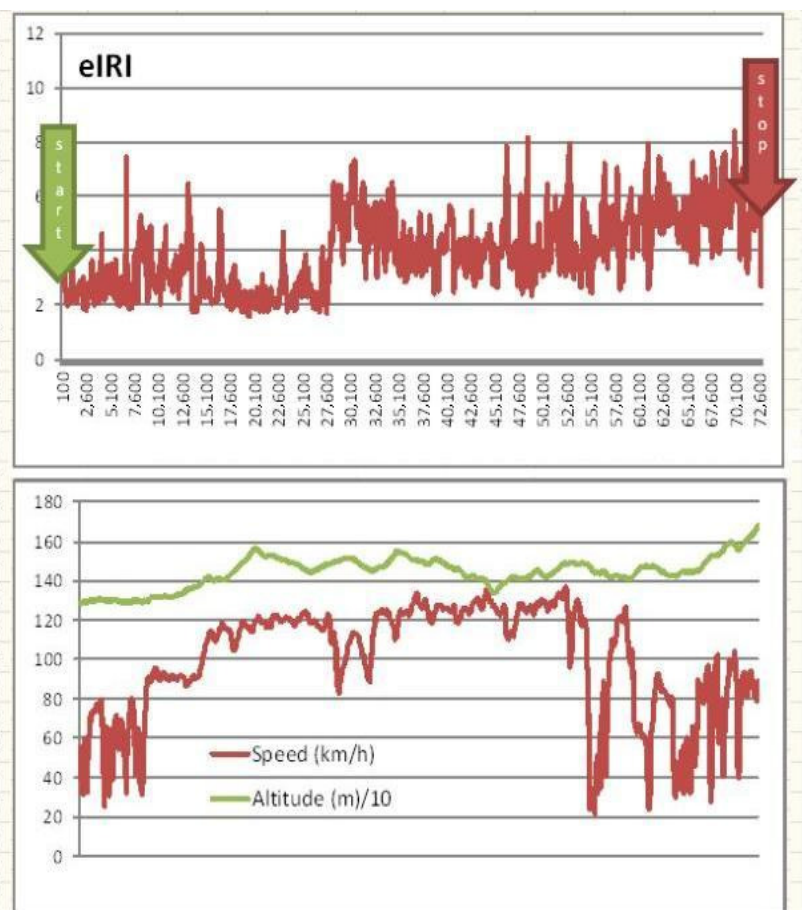

(b)

Fig. 8 From a $72 \mathrm{~km}$ test run in South Africa: (a) obtained measurement data which are sampled on a second level basis are averaged and aggregated into $100 \mathrm{~m}$ chunks; (b) the resulting data are then plotted in diagrams of eIRI, speed and altitude. Source: Roadroid internal documents. 
research, cIRI has been enabled in the application. Tests have been done which confirms that cIRI, if calibrated correctly, can meet the demands for road condition measurements. However, one needs to maintain a stable speed at around $60 \sim 80 \mathrm{~km} / \mathrm{h}$ for cIRI to work correctly. The quarter-car model uses two swinging weights which can be simulated if the vehicle chassis movement is available as input. The estimation of the chassis movement is based on the accelerometer data and a vehicle calibration variable, which is adjusted between $0.5 \sim 4$, in small steps by the Roadroid operator. Validation of sample data and the IRI output has been made with the ProVal (Profile Viewing and Analysis) [12] software. ProVal is the most widely-used, validated and reliable tool for pavement profile analysis according to the developers (The Transtec Group) and their US clients-FHWA (Federal Highway Administration) and the LTPP (Long Term Pavement Performance Program). We have however seen limited IRI-correlation on roads with rough surfaces (as chip seals/brick roads), but at the same time, promising results on gravel roads in Afghanistan and Sweden. From what we have seen, it is mainly a question of filtering the data correctly to get the correct device motion. Further work needs to be done in this field. As mobile technologies and sensors get more advanced, suitable distance measuring devices for distance monitoring between the road and chassis might be used in the future.

\section{Data Aggregator}

The needs are very different-from operational maintenance on developed road networks to first fact finding in developing countries. Our internet map solution is a good way to view data, but demands some basic knowledge of GIS and road databases.

We also developed a data aggregator which is able to aggregate and average date/time, coordinates (latitude, longitude), vertical profile (altitude) and speed together with eIRI and cIRI in selectable section lengths of 25 1,000 m (Fig. 8). In the current version, CSV files can be generated and imported into other software as digital spreadsheets or RMMS (road maintenance management systems).

\section{Professional Use 2013-2014}

Large-scale collection of measurement data in Sweden has been taking place during a 5 month period by the Swedish automobile association (Motormännen) [13]. The organization will sample $92,000 \mathrm{~km}$ of the Swedish road network to identify and point out road defects. We will prepare a report to Motormännen when the project is finished and point out where the worst roads in Sweden are. The project is financed by the Swedish Transport Administration (Trafikverket) [14] which is the Swedish government agency responsible for the long-term planning of the transport system.

\section{Use of the Built-in Camera}

As most smart phones now have a high quality built-in camera and GPS, we have developed a function to easily take localized photos and position them on the map (Fig. 9). The images are often of acceptable quality, but are subject to mounting and light conditions.

This is recognized as a very good support for visual inspections, and can also be used to capture dynamic events, such as certain snow conditions or other maintenance contract issues. We have also tested a high resolution, GPS data action video camera (Contour+2) [15] with good results for more precise and demanding video requirements.

\section{Use on Bicycle Pathways}

The cities of the world are facing more and more traffic problems. Cycling is one option to commute 


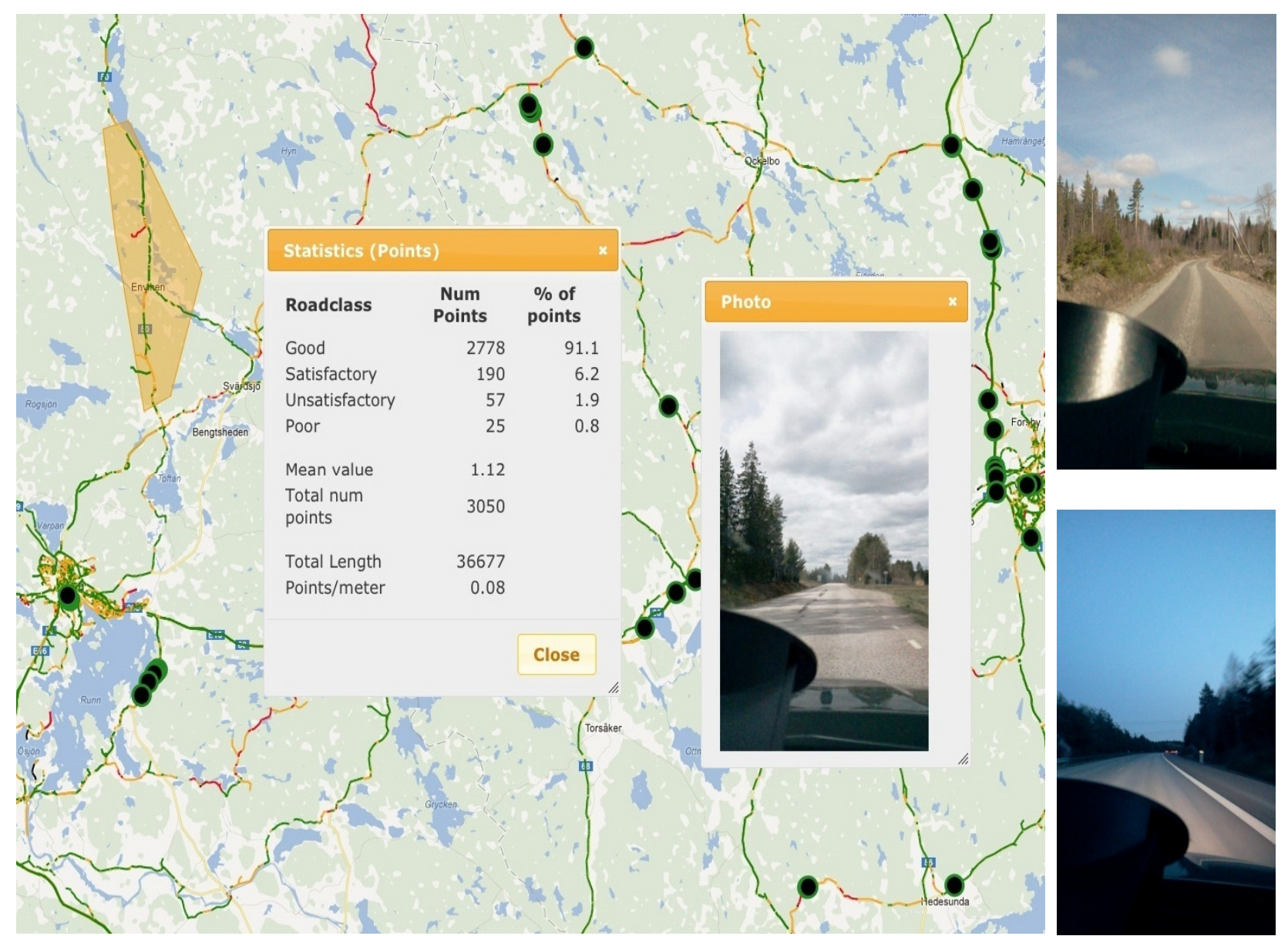

Fig. 9 Picture examples of the smart phones built-in camera and support in the web tool.

Source: www.roadroid.com.

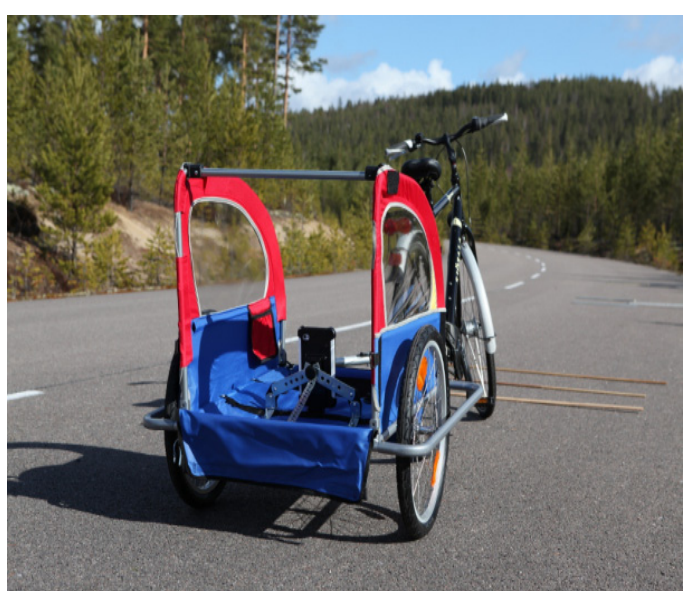

(a)

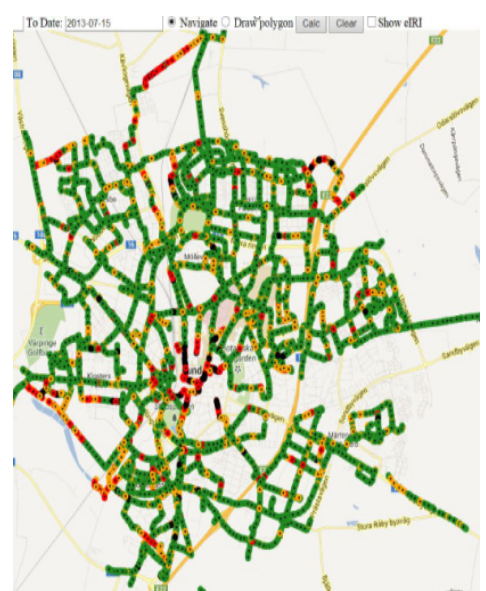

(b)

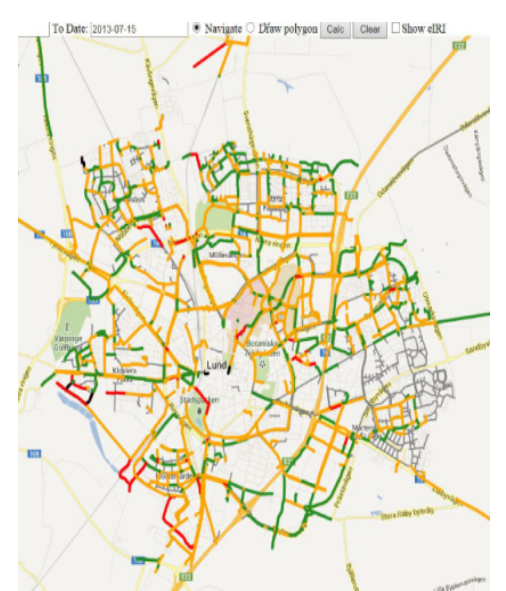

(c)

Fig. 10 Bike path condition monitoring: (a) Roadroid bicycle trailer mounting for bicycle pathways; (b) collected data examples using individual sampled dots; (c) aggregated and averaged road measurements links.

Source: Roadroid internal documents and www.roadroid.com.

instead of being stuck in traffic jams. The primary need for cyclists is safe cycle roads but also ride comfort.
One important parameter to accomplish better safety and ride comfort is the quality of the road surface. The 
same smart phone based system can be used as a quick and easy quality monitoring system for the cycle roads.

Since there is little or none previous work in this field and established road condition standards as IRI are absent, we use our own road class standard. To get dependable and reliable results, one needs to use an approved bicycle trailer (Fig. 10a). On the wheels axle, one can mount the Roadroid device firmly and register all the relevant variables. Since many cities yet have not made any inventory of their cycle roads, one can use the latitude/longitude data as input into a GIS tool and create geometries for bicycle pathways (Figs. 10b and 10c).

\section{Conclusions}

Measuring roads with smart phones can provide an efficient, scalable, and cost-effective way for road organizations to deliver road condition data. In this paper, we have illustrated this with the use of our software bundle-Roadroid. The system which does not require a network connection during the data collection can geo locate data (unmatched or matched to existing roads) on a globally level with sufficient accuracy. We have implemented road condition standards based on previous work [1] as cIRI as well as our own speed and vehicle independent eIRI standard, which correlate up to $81 \%$ with laser measurement systems [10, 11]. On our clouds-based web GIS platform, one can use powerful filtering techniques as the RI for road maintenance decision support. Further work by examining and analyzing large data sets as well as incorporating new sensors can further improve the solution. By broadcasting road condition warnings through standards for ITS, the information could provide new kinds of dynamic and valuable input to automotive navigation systems and digital route guides for special traffic, etc..

\section{Acknowledgments}

The authors would like to thank the Kartographic
Society-Innovation Award, UN World Summit Award-Global Champion in eGovernance and European Satellite Navigation Competition-regional winner for their help.

\section{References}

[1] Sayers, M. W., Gillespie, T. D., and Queiroz, C. A. V. 1986. "The International Road Roughness Experiment: Establishing Correlation and a Calibration Standard for Measurements." World Bank technical paper.

[2] Sayers, M. W., and Karamihas, S. 1996. Interpretation of Road Roughness Profile Data. Final report of University of Michigan.

[3] Dana, H. J., Teller, L. W., Martin, G. E., and Bryant, C. B. 1932. "The Dana Automatic Recording Roughometer for Measuring Highway Roughness." In Proceedings of the 12th Annual Meeting of the Highway Research Board. Accessed May 30, 2014. http://trid.trb.org/view.aspx?id=105065.

[4] OpenLayers. 2014. “OpenLayers Home.” OpenLayers. Accessed May 30, 2014. http://openlayers.org/.

[5] OpenStreetMap. 2014.2 "OpenStreetMap." OpenStreetMap. Accessed May 30, 2014. http://www.openstreetmap.org/.

[6] Trafikverket. 2014. "Start Page-NVDB." Trafikverket. Accessed May 30, 2014. http://www.nvdb.se/.

[7] Sayers, M. W., Gillespie, T. D., and Paterson, W. D. O. 1986. "Guidelines for Conducting and Calibrating Road Roughness Measurements.” World Bank technical paper.

[8] Wakeham, K. J., and Rideout, D. G. 2011. "Model Complexity Requirements in Design of Half Car Active Suspension Controllers." Presented at ASME (American Society of Mechanical Engineers) 2011 Dynamic Systems and Control Conference and Bath/ASME Symposium on Fluid Power and Motion Control, Virginia.

[9] Tarr, K. E. 2013. "Evaluation of Response Type Application for Measuring Road Roughness." M.Sc. thesis, University of Pretoria, South Africa.

[10] Johnston, M. 2013. "Using Cell-Phones to Monitor Road Roughness." M.Sc. thesis, University of Auckland.

[11] Islam, T. 2013. "Using Cell-Phones to Monitor Road Roughness." M.Sc. thesis, University of Auckland.

[12] ProVal. 2014. "ProVal: View and Analyze Pavement Profiles.” ProVal. Accessed May 30, 2014. http://www.roadprofile.com/.

[13] Motormännen. 2014. "Vägombuden Kontrollerar Svenska Vägars Säkerhet (Road Inspectors Check Condition of 
Swedish Roads).” Motormännen. Accessed May 30, 2014. https://www.motormannen.se/nyheter/2014/vagombudenkontrollerar-svenska-vagars-sakerhet/. (in Swedish)

[14] Trafikverket. 2014. "Homepage." Trafikverket. Accessed
May 30, 2014. http://www.trafikverket.se/. (in Swedish)

[15] Contour. 2014. “Contour-Contour+2." Contour. Accessed May 30, 2014. http://contour.com/ collections/cameras/products/contour-2. 